OPEN ACCESS

Edited by:

Emanuele Azzoni,

University of Milano Bicocca, Italy

Reviewed by:

Majlinda Lako,

Newcastle University, United Kingdom

Dominic Owens,

University of Oxford, United Kingdom

*Correspondence:

Clara Bueno

cbueno@carrerasresearch.org

Pablo Menendez

pmenendez@carrerasresearch.org

Specialty section:

This article was submitted to

Stem Cell Research,

a section of the journal

Frontiers in Cell and Developmental

Biology

Received: 01 December 2020

Accepted: 09 April 2021

Published: 21 May 2021

Citation:

Gutierrez-Agüera $F$

Rodriguez-Cortez V, Petazzi P,

Bueno $C$ and Menendez $P$ (2021) A Benchmark Side-by-Side Comparison of Two Well-Established Protocols for in vitro Hematopoietic Differentiation From Human

Pluripotent Stem Cells.

Front. Cell Dev. Biol. 9:636704. doi: 10.3389/fcell.2021.636704

\section{A Benchmark Side-by-Side Comparison of Two Well-Established Protocols for in vitro Hematopoietic Differentiation From Human Pluripotent Stem Cells}

\author{
Francisco Gutierrez-Agüera', Virginia Rodriguez-Cortez ${ }^{1}$, Paolo Petazzi', \\ Clara Bueno ${ }^{1,2 *}$ and Pablo Menendez ${ }^{1,2,3 *}$
}

1 Josep Carreras Research Institute, Barcelona, Spain, ${ }^{2}$ Centro de Investigación Biomédica en Red de Cáncer (CIBER-ONC), Instituto de Salud Carlos III (ISCIII), Barcelona, Spain, ${ }^{3}$ Instituciò Catalana de Recerca i Estudis Avançats (ICREA), Barcelona, Spain

The generation of transplantable hematopoietic stem cells (HSCs) from human pluripotent stem cells (hPSCs) remains challenging. Current differentiation protocols from hPSCs generate mostly hematopoietic progenitors of the primitive HSCindependent program, and it remains unclear what is the best combination of cytokines and hematopoietic growth factors (HGFs) for obtaining functional hematopoietic cells in vitro. Here, we have used the AND1 and H9 hESC lines and the H9:dualreporter RUNX1C-GFP-SOX17-Cherry to compare the hematopoietic differentiation in vitro based on the treatment of embryoid bodies (EBs) with the ventral mesoderm inducer BMP4 plus HGFs in the absence (protocol 1) or presence (protocol 2) of stage-specific activation of Wnt/ $\beta$-catenin and inhibition of Activin/Nodal. Despite a slight trend in favor of protocol 1, no statistically significant differences were observed between protocols at any time point analyzed throughout EB development regarding the frequency of hemogenic endothelial (HE) precursors; CD43+ CD45-, CD45+, and CD45 + CD34 + hematopoietic derivatives; or the output of clonogenic progenitors. Similarly, the kinetics of emergence throughout EB development of both SOX17 + HE and RUNX1C + definitive hematopoiesis was very similar for both protocols. The expression of the early master mesendodermal transcription factors Brachyury, MIXL1, and KDR revealed similar gene expression kinetics prior to the emergence of RUNX1C + definitive hematopoiesis for both protocols. Collectively, the simpler protocol 1 is, at least, as efficient as protocol 2 , suggesting that supplementation with additional morphogens/HGFs and modulation of Activin/Nodal and Wnt/ $\beta$-catenin pathways seem dispensable for in vitro hematopoietic differentiation of hPSCs.

Keywords: HPSC, hematopoiesis, differentiation, cytokines, WNT/B-CATENIN 


\section{INTRODUCTON}

Directed differentiation of human pluripotent stem cells (hPSCs) into specific cell types would enable the generation of large numbers of patient- or donor-derived cells for regenerative medicine and the implementation of unique in vitro models for studying developmental biology, disease modeling, and drug screening (Menendez et al., 2006). In the hematopoietic setting, the generation of transplantable hematopoietic stem cells (HSCs) from hPSCs remains challenging because both the primitive and definitive developmental programs are intermingled, and current hPSC differentiation protocols generate mostly hematopoietic progenitors of the primitive HSC-independent program (Medvinsky et al., 2011). However, multiple studies have reported the generation of distinct hematopoietic cell types from hPSCs in vitro, either by co-culturing them with stromal cell layers such as OP9 cells or by directing their differentiation through embryoid body (EB) development with specific morphogens and hematopoietic growth factors (HGFs) (Demirci et al., 2020).

Hematopoietic development from hPSCs arises from early VEGF receptor (KDR) + CD34-CD31- mesodermal progenitors, and it transitions through $\mathrm{CD} 34+\mathrm{CD} 31+\mathrm{CD} 45-$ hemogenic endothelium (HE) precursors (Chadwick et al., 2003; Wang et al., 2004). The expression of glycophorin A (CD235) is used as a surrogate marker to identify the primitive $(\mathrm{CD} 235+)$ or definitive $(\mathrm{CD} 235-)$ hematopoietic potential (Sturgeon et al., 2014). HE precursors further differentiate toward CD43 + CD45- and then CD45 + hematopoietic cells (Chadwick et al., 2003; Menendez et al., 2004; Wang et al., 2004, 2005). Early protocols for the successful EB-based in vitro-directed differentiation of hPSCs into hematopoietic cells relied on the use of the master early ventral mesoderm inducer BMP4 and different cocktails of HGFs including the early acting hematopoietic cytokines SCF and FLT3L (Chadwick et al., 2003; Wang et al., 2004, 2005; Ledran et al., 2008; Ditadi et al., 2017). Further studies over the last decade suggested that Activin/Nodal and Wnt/ $\beta$-catenin pathways regulate primitive vs. definitive in vitro hematopoietic specification from hPSCs (Sturgeon et al., 2014; Ditadi and Sturgeon, 2016; Ditadi et al., 2017). These studies suggest that the specification of definitive hematopoiesis requires early stage-specific activation of $\mathrm{Wnt} / \beta$-catenin and inhibition of Activin/Nodal signaling pathways, which is efficiently achieved by treatment with the GSK-3 inhibitor CHIR99021, a Wnt agonist, and the Activin/Nodal inhibitor SB-431542, respectively (Bendall et al., 2007; Kennedy et al., 2012). Although many studies have investigated early hematopoietic development by interrogating the role of instructive transcription factors, it remains unclear what is the best combination of morphogens, cytokines, and HGFs to be used for obtaining functional hematopoietic cells in vitro. Here, we have compared the hematopoietic differentiation in vitro of two well-established protocols which rely on EB treatment with BMP4 plus a different cocktail HGFs in the absence or presence of stage-specific activation of $\mathrm{Wnt} / \beta$-catenin and inhibition of Activin/Nodal.

\section{MATERIALS AND METHODS}

\section{Maintenance of hPSC Lines}

Human embryonic stem cell (hESC) lines, including the dual reporter SOX17 $7^{m C H E R R Y / W}$ RUNX1C $C^{G F P / W}$ H9 cells [kindly provided by Prof. Andrew Elefanty (Murdoch Children's Research Institute, Monash University, VIC, Australia) and Dr. Andrea Ditadi (Ospedale San Raffaelo, Milan, Italy)], were maintained undifferentiated in T25 flasks on a layer of irradiated murine embryonic fibroblasts in complete Dulbecco's modified Eagle's medium (DMEM) containing 20\% knockout (KO) serum replacement and $8 \mathrm{ng} / \mathrm{ml}$ basic fibroblast growth factor (bFGF) as extensively described (Chadwick et al., 2003; Ramos-Mejia et al., 2014; Bueno et al., 2019). The medium was changed daily and cells were passaged weekly by dissociation with 1:1 collagenase type IV:dispase (Invitrogen, Carlsbad, CA, United States). Cultures were visualized daily by phase contrast microscopy. Approval for the hESC work was obtained from our local health authorities and the Spanish National Pluripotent Ethical Committee (0336E/14973/2017).

\section{Hematopoietic Differentiation From hPSCs by EB Formation}

On the day of passage, undifferentiated hESCs at confluence in T25 culture flasks $\left(\sim 8 \times 10^{6}\right.$ alive cells $)$ were first treated with collagenase type IV:dispase for $1 \mathrm{~h}$ at $37^{\circ} \mathrm{C}$, and dispersed cells were transferred to six-well low-attachment plates $\left(\sim 1 \times 10^{6}\right.$ alive cells/well/condition; alive cells were measured by trypan blue exclusion) and incubated overnight in differentiation medium (DM; KO-DMEM supplemented with $20 \%$ fetal bovine serum, $1 \%$ non-essential amino acids, $1 \mathrm{mmol} / \mathrm{L} \mathrm{L}$-glutamine, and $0.1 \mathrm{mmol} / \mathrm{L} \beta$-mercaptoethanol). Media changes and supplementation with BMP4, different HGFs, and inhibitors were performed as in Figure 1A. Concentrations used were as follows: $3 \mu \mathrm{M}$ CHIR99021, $3 \mu \mathrm{M}$ SB-431542, $25 \mathrm{ng} / \mathrm{ml} \mathrm{BMP4,} 300 \mathrm{ng} / \mathrm{ml}$ stem cell factor (SCF), $300 \mathrm{ng} / \mathrm{ml}$ FMS-like tyrosine kinase 3 ligand (Flt3L), $10 \mathrm{ng} / \mathrm{ml}$ interleukin (IL)-3, $10 \mathrm{ng} / \mathrm{ml} \mathrm{IL-6,50} \mathrm{ng/ml} \mathrm{granulocyte-colony} \mathrm{stimulating}$ factor (G-CSF), $15 \mathrm{ng} / \mathrm{ml}$ VEGF, $10 \mathrm{ng} / \mathrm{ml}$ basic fibroblast growth factor 2 (FGF2), $25 \mathrm{ng} / \mathrm{ml}$ insulin-like growth factor-1 (IGF1), $30 \mathrm{ng} / \mathrm{ml}$ thrombopoietin (TPO), and two IU erythropoietin (EPO) (all from R\&D Systems, Minneapolis, MN, United States) (Chadwick et al., 2003; Sturgeon et al., 2014; Ditadi and Sturgeon, 2016). In the serum-free experiments, the basal media SFD composed of IMDM and Ham's F12 (Gibco) supplemented with L-glutamine $(2 \mathrm{mM})$, ascorbic acid $(1 \mathrm{mM})$, monothioglycerol (MTG, $4 \times 10^{-4} \mathrm{M}$; Sigma), transferrin (150 $\left.\mu \mathrm{g} / \mathrm{ml}\right), \mathrm{N} 2(100 \times)$, B27 $(100 \times)$, and bovine serum albumin (BSA) $0.1 \%$ was used.

Embryoid bodies were dissociated at different time points during development using collagenase B and enzyme-free cell dissociation buffer (Invitrogen). Dissociated cells were stained with anti-CD34-PE, anti-CD31-FITC, anti-CD45-APC or anti-CD34-PE-Cy7, CD31-BV510, anti-glycophorin A-PE, antiCD43-FITC, and anti-CD45-APC antibodies and 7-actinomycin $\mathrm{D}$ (7AAD) for exclusion of dead cells and analyzed using a FACS Canto flow cytometer (BD Biosciences). The emergence of alive 

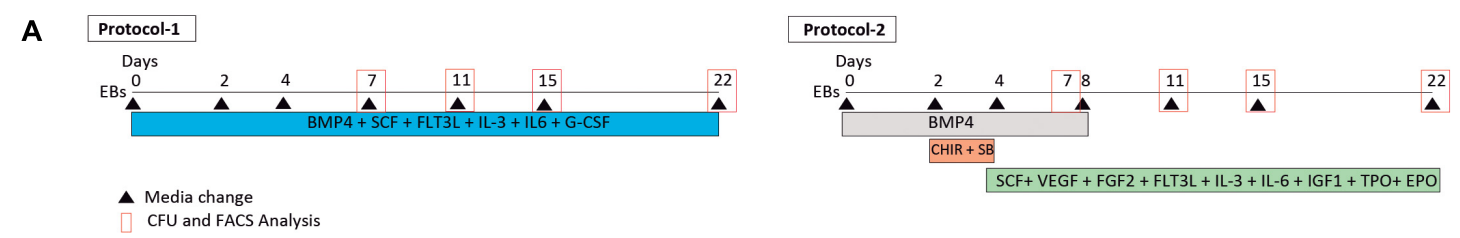

Media change

B

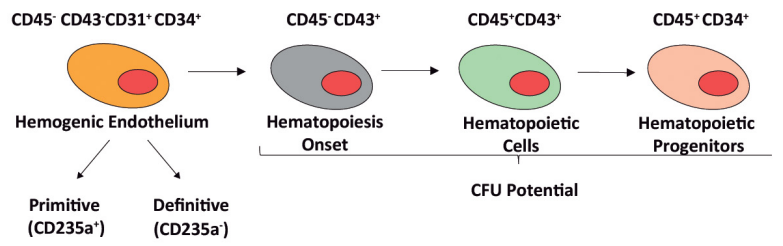

C
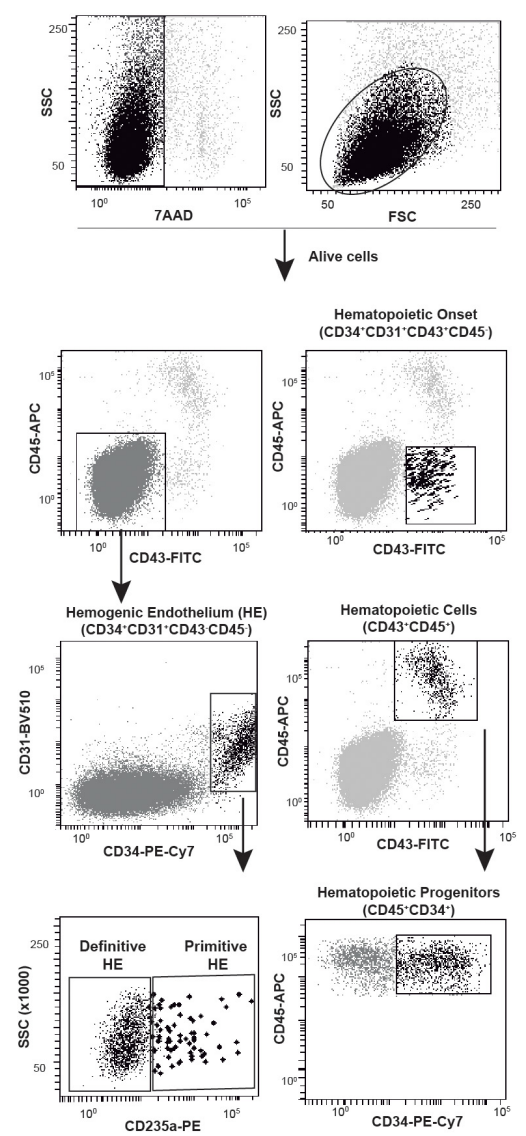

D
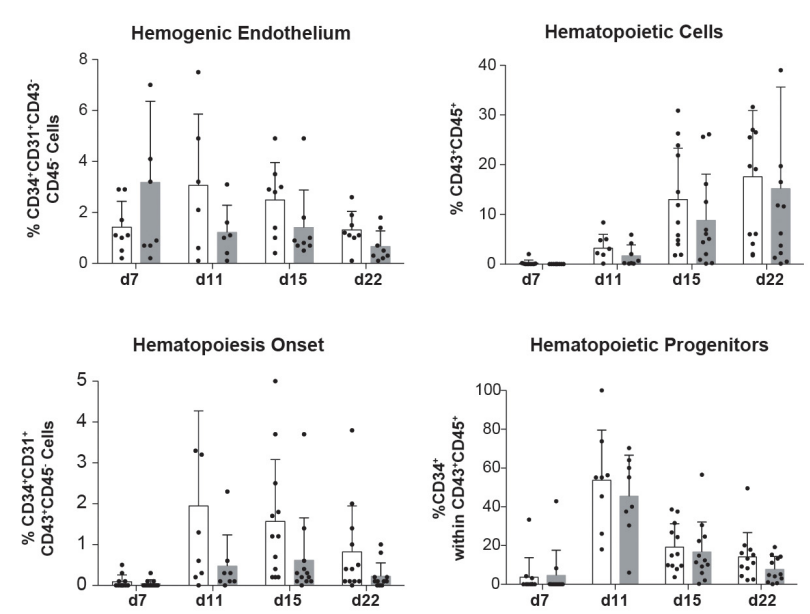

E

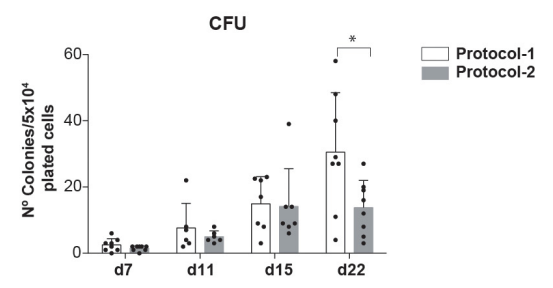

FIGURE 1 | Side-by-side comparison of two well-established EB-based directed hematopoietic differentiation protocols. (A) Schematic of the differentiation protocols (protocol 1 and protocol 2) and time-point analyses. (B) Cartoon depicting the hematopoietic cell fate specification through the formation of hEBs. (C) Representative FACS analysis and identification of the indicated alive hematopoietic cell populations analyzed during hematopoietic commitment of hPSCs. (D) Frequency of alive (7AAD-) HE precursors (CD45-CD43-CD34 + CD31 +), CD43 + CD45- and CD45 + hematopoietic cells, and hematopoietic progenitors $(\mathrm{CD} 45+\mathrm{CD} 34+)$ analyzed at the indicated time points throughout EB development $(n=8)$. (E) Clonogenic progenitors detected by hematopoietic CFU assays were analyzed at the indicated time points $(n=8)$. Each individual dot represents the value for each independent in vitro differentiation experiment. Data were plotted as mean \pm SD. n.s., not significant. * $p$ value $<0.05$.

(7AAD-) SOX17 + and RUNX1C + cells during EB development was analyzed using Cherry and GFP reporters, respectively. Colony-forming unit (CFU) assays were performed at different time points along EB differentiation by plating $5 \times 10^{4} \mathrm{~EB}-$ derived cells onto serum-free methylcellulose H4435 (Stem Cell Technologies, Vancouver, BC, Canada). Colonies were scored 
after 12 days using standard CFU scoring assays (Chadwick et al., 2003; Ramos-Mejia et al., 2014; Bueno et al., 2019).

\section{Real-Time Reverse Transcriptase-Polymerase Chain Reaction}

DNAse-treated total RNA was extracted from EBs using the Maxwell RSC simplyRNA Cells Kit (Promega, Madison, WI, United States). Two hundred and fifty nanograms of RNA was retrotranscribed with random hexamers using the Superscript III first-strand synthesis kit (Thermo Fisher Scientific, Waltham, MA, United States). The resulting cDNA was diluted 1:2 and analyzed for differential gene expression using the PowerUp SYBR Green Master Mix (Thermo Fisher Scientific, Waltham, MA, United States) on a Bio-Rad CFX384 qPCR Platform (Bio-Rad, Hercules, CA, United States). Relative expression of the mesoderm markers was calculated with the $\Delta \Delta$ Ct method using RPL19 as the housekeeping gene. Primer sequences were as follows: KDR: Fw-CCACTGGTATTGGCAGTTGGA, RevCACAAGGGTATGGGTTTGTCAACT (80 bp, exons 10-11); T/BRACHYURY: Fw-ATGAGCCTCGAATCCACATAGT, Rev-TCCTCGTTCTGATAAGCAGTCA (108 bp, exon 3); MIXL1: Fw-GGATCCAGGTATGGTTCCAG, RevGGAGCACAGTGGTTGAGGAT (130 bp, exons 1-2); and RPL19: Fw-GCGGAAGGGTACAGCCAAT, RevAGCAGCCGGCGCAAAATCC (78 bp, exon 4). The absence of genomic DNA contamination was confirmed by running RT controls.

\section{Statistical Analyses}

Data are plotted as mean \pm standard deviation (SD). $p$ values were calculated using paired two-tailed Student's $t$ test for each time point using the Prism software version 8.0 (GraphPad Prism Software Inc., San Diego, CA, United States).

\section{RESULTS}

We have compared whether two extensively employed EBbased directed differentiation protocols influence hPSC-derived hematopoietic differentiation in vitro. The differentiation protocols exclusively differed in the cocktail of morphogens and HGFs used during EB development (Figure 1A). The simpler protocol was developed by Bhatia's lab in 2003 and used the ventral mesoderm inducer BMP4 plus the HGFs SCF, FLT3L, IL-3, IL-6, and G-CSF throughout the entire 22-day differentiation protocol (Chadwick et al., 2003; Menendez et al., 2004; Wang et al., 2004, 2005) (termed as protocol 1, Figure 1A). The other differentiation protocol was developed later on by Keller's lab and employed a more complex setup of morphogens and HGFs suggested to more efficiently promote EB differentiation toward the definitive hematopoietic program (Kennedy et al., 2012; Ditadi and Sturgeon, 2016; Ditadi et al., 2017). It includes an 8-day treatment with BMP4 and a concomitant stage-specific (day 2 to day 4 of EB development) Wnt/ $\beta$-catenin activation with the GSK-3 inhibitor CHIR99021 and Activin/Nodal inhibition with the inhibitor SB-431542 followed by treatment with SCF, VEGF, FGF2, FLT3L, IL-3, IL-6, IGF1, TPO, and EPO from day 4 of EB development onward (termed as protocol 2, Figure 1A). The in vitro efficiency of both protocol 1 and protocol 2 was compared side-by-side using the hESC lines AND1 and H9 ( $n=8$ independent experiments). The frequency of HE precursors (CD45-CD43-CD34 + CD31+), hematopoietic cells (both CD43 + CD45- and CD43 + CD45+), and hematopoietic progenitors $(\mathrm{CD} 45+\mathrm{CD} 34+)$ as well as the output of clonogenic progenitors detected by hematopoietic CFU assays was analyzed at indicated time points throughout EB development (Figures 1B-E). Overall, although a slight trend in favor of protocol 1 was consistently observed, no statistically significant differences were observed in the frequency of either HE precursors; CD43 + CD45-, CD45+, or $\mathrm{CD} 45+\mathrm{CD} 34$ + hematopoietic derivatives (Figure 1D); or the output of clonogenic progenitors (Figure 1E) at any time point analyzed (from day 7 to day 22 of EB development). Of note, $\sim 95 \%$ of the HE precursors were CD235- regardless of the protocol employed (Figure 1C).

We next took advantage of the RUNX1C-GFP-SOX17-Cherry dual reporter H9 hESC line to track the endothelial $($ SOX17 +) to definitive hematopoietic (RUNX1C+) transition (Ng et al., 2016). Our data showed that the protocol employed (protocol 1 vs protocol 2) had no impact on the kinetics of emergence of either SOX17 + HE or RUNX1C + definitive hematopoiesis throughout the 22 days of EB development ( $n=4$, Figures $2 A, \mathbf{B})$. The limited impact of the early stage-specific activation of $\mathrm{Wnt} / \beta$-catenin and inhibition of Activin/Nodal pathways in definitive hematopoietic development in vitro was further confirmed at the molecular level. Real-time reverse transcriptase-PCR-based expression of the early master mesendodermal transcription factors Brachyury, MIXL1, and KDR revealed very similar gene expression kinetics prior to the emergence of RUNX1C + definitive hematopoiesis ( $n=4$, Figure 2C).

Protocol 1 has been historically used in the presence of serum and protocol 2 in the absence of serum. To assess whether the presence of serum masks the differences between both protocols, the RUNX1C-GFP-SOX17-Cherry H9 hESC line was differentiated $(n=2)$ for 22 days in serum-free medium with the morphogens and HGFs of protocol 1 vs. protocol 2. As shown in Figures 2D-E, no significant differences were observed either in frequencies or in absolute numbers between protocols for either RUNX1C + or SOX17 + cells, indicating that the lack of significant differences in the hematopoietic output between both protocols is not attributable to (masked by) the presence of serum. Collectively, our in vitro data using different hESC lines demonstrate that the simpler protocol 1 is, at least, as efficient as protocol 2, suggesting that the supplementation with additional morphogens/HGFs and modulation of Activin/Nodal and $\mathrm{Wnt} / \beta$-catenin pathways seem dispensable for in vitro hematopoietic differentiation of hPSCs.

\section{DISCUSSION}

Human PSCs have long been postulated as an unprecedented model for studies on human development and disease modeling 
A

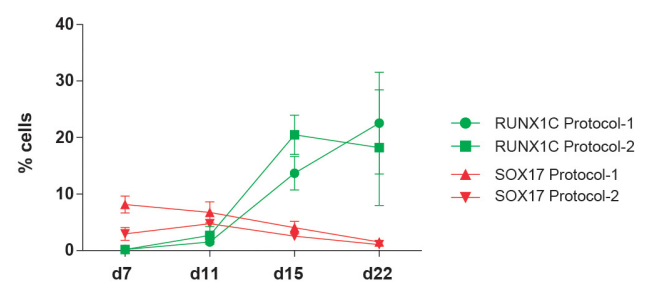

B

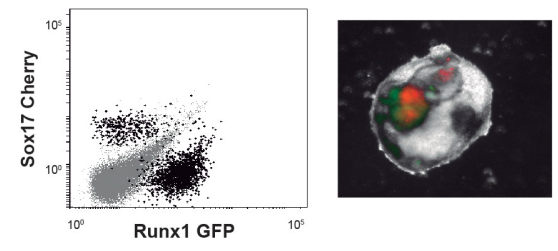

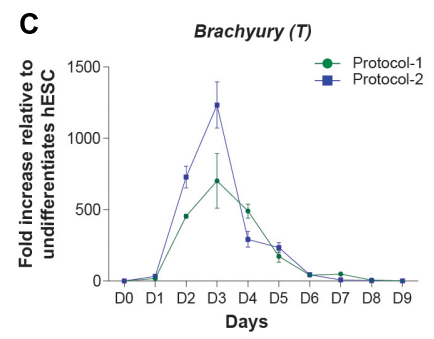

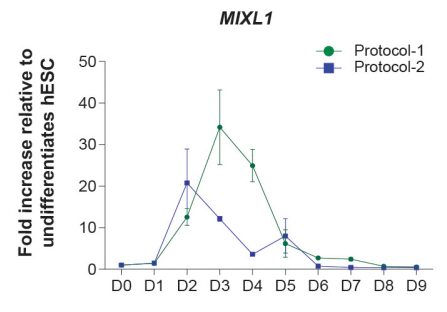

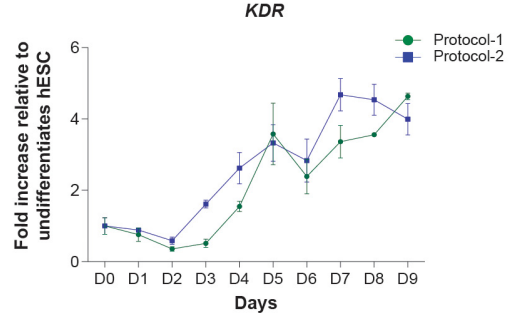

Days
D

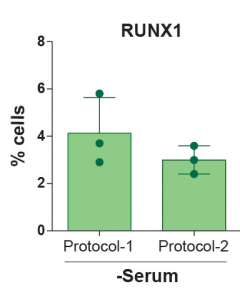

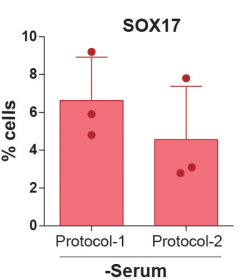
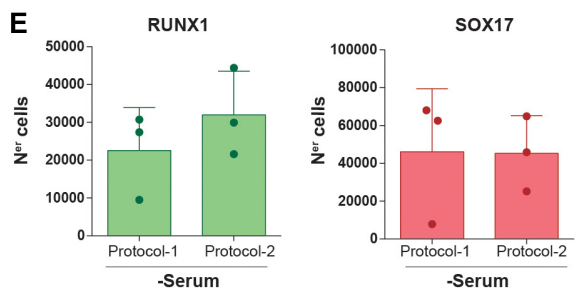

FIGURE 2 | Impact of both differentiation protocols on the emergence kinetics of SOX17 + HE and RUNX1C + definitive hematopoietic cells. (A) Frequency of alive (7AAD-) SOX17:Cherry + and RUNX1C:GFP + cells along EB differentiation with protocol 1 and protocol $2(n=4)$. (B) Left panel, representative flow cytometry plots displaying how (SOX17/Cherry + ) and definitive hematopoietic cells (RUNX1/GFP +) are identified. The right panel shows mCherry and GFP expression within differentiating EBs by fluorescence microscopy. (C) Gene expression kinetics of the mesendodermal transcription factors Brachyury (T), MIXL1, and KDR during mesoderm induction and early hematopoietic differentiation with protocol 1 and protocol $2(n=4)$. (D,E) Frequency (D) and absolute numbers (E) of RUNX1/GFP + definitive hematopoietic cells and SOX17/Cherry + HE cells at endpoint (day 22) of in vitro differentiation using protocol 1 or protocol 2 in a serum-free media. Data were plotted as mean \pm SD. n.s., not significant.

and for drug testing (Menendez et al., 2006). Many studies have investigated early hematopoietic development by interrogating the role of instructive transcription factors (Real et al., 2012, 2013; Ramos-Mejia et al., 2014; Ayllón et al., 2015; Bueno et al., 2019). Nonetheless, it remains unclear regarding the best combination of cytokines and growth factors, the timing of treatment, and the methodology to be used for obtaining fully functional hematopoietic cells in vitro. There are a large number of published in vitro protocols for differentiation of hPSCs toward hematopoietic cells. These protocols largely differ in many aspects including (i) the use of several EB systems (spin and non-spin EBs) vs. stromal cells such as OP9, MSCs, AGM feeders, fetal liver feeders, etc.; (ii) the absence or presence of sera from different mammalian sources; and (iii) a never-ending combination of morphogens and HGFs, among others. However, none of the protocols used so far render hPSC hematopoietic derivatives capable of reconstituting the hematopoietic system in immune-deficient mice.
Two well-established, widely used in vitro hematopoietic differentiation protocols exist. The first protocol, initially developed by Bhatia's lab (termed as protocol 1 in this study), relies on EB treatment with the ventral mesoderm inducer BMP4 together with the HGFs SCF, FLT3L, IL-3, IL-6, and G-CSF for further blood specification for 22 days (Chadwick et al., 2003; Menendez et al., 2004; Wang et al., 2004, 2005). The other protocol developed by Keller's lab employs BMP4 together with a concomitant stage-specific (days 2-4 of EB development) Wnt $/ \beta$ catenin activation with the GSK-3 inhibitor CHIR99021 and Activin/Nodal inhibition with the inhibitor SB-431542 followed by treatment with SCF, VEGF, FGF2, FLT3L, IL-3, IL-6, IGF1, TPO, and EPO from day 4 of EB development onward (termed as protocol 2 in this study) (Kennedy et al., 2012; Sturgeon et al., 2014; Ditadi and Sturgeon, 2016; Ditadi et al., 2017). The GSK-3 inhibitor CHIR99021 and the Activin/Nodal inhibitor SB-431542 were selected based on previous reports showing that WNT3A exposure during mesoderm patterning of hESCs suppresses primitive hematopoiesis (Gertow et al., 2013) and that WNT agonists or ACTIVIN antagonists may support definitive 
hematopoiesis from hPSCs (Kennedy et al., 2012; Sturgeon et al., 2014). Both inhibitors are used between day 2 and day 4 of EB differentiation, the period during which HOX gene expression is initiated (Ramos-Mejia et al., 2014; Ng et al., 2016).

In this study, we have used several wild-type and reporter hPSC lines to compare the hematopoietic differentiation in vitro based on the treatment of EBs with BMP4 plus HGFs in the absence (protocol 1) or presence (protocol 2) of stage-specific activation of $\mathrm{Wnt} / \beta$-catenin and inhibition of Activin/Nodal. We demonstrate that the simpler protocol 1 is, at least, as efficient as protocol 2, suggesting that the supplementation with additional morphogens/HGFs and modulation of Activin/Nodal and Wnt/ $\beta$-catenin pathways seem dispensable for yielding a higher number of hematopoietic (progenitor) derivatives for subsequent downstream in vitro applications. Furthermore, preliminary data suggest that a similar trend of hematopoietic differentiation was observed in serum-free conditions indicating that the lack of significant differences in the hematopoietic output between both protocols is not masked by the presence of serum. Further side-by-side in vitro comparisons should comprehensively investigate the hematopoietic output of the multiple combinations of morphogens and HGFs used in parallel or sequentially in EBbased hematopoietic differentiation protocols reported from multiple laboratories.

\section{DATA AVAILABILITY STATEMENT}

The raw data supporting the conclusions of this article will be made available by the authors, without undue reservation.

\section{REFERENCES}

Ayllón, V., Bueno, C., Ramos-Mejía, V., Navarro-Montero, O., Prieto, C., Real, P. J., et al. (2015). The Notch ligand DLL4 specifically marks human hematoendothelial progenitors and regulates their hematopoietic fate. Leukemia 29, 1741-1753. doi: 10.1038/leu.2015.74

Bendall, S. C., Stewart, M. H., Menendez, P., George, D., Vijayaragavan, K., Werbowetski-Ogilvie, T., et al. (2007). IGF and FGF cooperatively establish the regulatory stem cell niche of pluripotent human cells in vitro. Nature 448, 1015-1021. doi: 10.1038/nature06027

Bueno, C., Calero-Nieto, F. J., Wang, X., Valdes-Mas, R., Gutierrez-Aguera, F., Roca-Ho, H., et al. (2019). Enhanced hemato-endothelial specification during human embryonic differentiation through developmental cooperation between AF4-MLL and MLL-AF4 fusions. Haematologica 104, 1189-1201. doi: 10.3324/ haematol.2018.202044

Chadwick, K., Wang, L., Li, L., Menendez, P., Murdoch, B., Rouleau, A., et al. (2003). Cytokines and BMP-4 promote hematopoietic differentiation of human embryonic stem cells. Blood 102, 906-915. doi: 10.1182/blood-2003-03-0832

Demirci, S., Leonard, A., and Tisdale, J. F. (2020). Hematopoietic stem cells from pluripotent stem cells: Clinical potential, challenges, and future perspectives. Stem Cells Transl. Med. 9, 1549-1557. doi: 10.1002/sctm.20-0247

Ditadi, A., and Sturgeon, C. M. (2016). Directed differentiation of definitive hemogenic endothelium and hematopoietic progenitors from human pluripotent stem cells. Methods 101, 65-72. doi: 10.1016/j.ymeth.2015.10.001

Ditadi, A., Sturgeon, C. M., and Keller, G. (2017). A view of human haematopoietic development from the Petri dish. Nat. Rev. Mol. Cell. Biol. 18, 56-67. doi: $10.1038 / \mathrm{nrm} .2016 .127$

\section{ETHICS STATEMENT}

Approval for hESC work was obtained from the ISCIII-Comisión Nacional de Garantías (0336E/14973/2017).

\section{AUTHOR CONTRIBUTIONS}

FG-A designed and performed the experiments and analyzed the data. VR-C and PP performed the experiments. CB and PM conceived the study, designed the experiments, analyzed the data, and wrote the manuscript. All authors contributed to the article and approved the submitted version.

\section{FUNDING}

We thank CERCA/Generalitat de Catalunya and Fundació Josep Carreras-Obra Social la Caixa for institutional support. Financial support for this work was obtained from the Generalitat de Catalunya (SGR180, PERIS 2017-2019) and the Spanish Ministry of Economy and Competitiveness (RTC2018-4603-1) to PM. $\mathrm{PM}$ is an investigator of the Spanish Cell Therapy cooperative network (TERCEL). CB acknowledges financial support from Health Institute Carlos III (PI20/00822).

\section{ACKNOWLEDGMENTS}

We would like to thank Andrea Ditadi (Ospedale San Raffaelo, Milan, Italy) and Prof. Andrew Elefanty (Murdoch Children's Research Institute and Monash University, VIC, Australia) for sharing their H9:SOX17-Cherry-RUNX1C-GFP cell line.

Gertow, K., Hirst, C. E., Yu, Q. C., Ng, E. S., Pereira, L. A., Davis, R. P., et al. (2013). WNT3A promotes hematopoietic or mesenchymal differentiation from hESCs depending on the time of exposure. Stem Cell Rep. 1, 53-65. doi: 10.1016/j. stemcr.2013.04.002

Kennedy, M., Awong, G., Sturgeon, C. M., Ditadi, A., LaMotte-Mohs, R., ZunigaPflucker, J. C., et al. (2012). T lymphocyte potential marks the emergence of definitive hematopoietic progenitors in human pluripotent stem cell differentiation cultures. Cell Rep. 2, 1722-1735. doi: 10.1016/j.celrep.2012.11. 003

Ledran, M. H., Krassowska, A., Armstrong, L., Dimmick, I., Renstrom, J., Lang, R., et al. (2008). Efficient hematopoietic differentiation of human embryonic stem cells on stromal cells derived from hematopoietic niches. Cell Stem Cell 3, 85-98. doi: 10.1016/j.stem.2008.06.001

Medvinsky, A., Rybtsov, S., and Taoudi, S. (2011). Embryonic origin of the adult hematopoietic system: advances and questions. Development 138, 1017-1031. doi: 10.1242/dev.040998

Menendez, P., Bueno, C., and Wang, L. (2006). Human embryonic stem cells: A journey beyond cell replacement therapies. Cytotherapy 8, 530-541. doi: 10.1080/14653240601026654

Menendez, P., Wang, L., Chadwick, K., Li, L., and Bhatia, M. (2004). Retroviral transduction of hematopoietic cells differentiated from human embryonic stem cell-derived CD45(neg)PFV hemogenic precursors. Mol. Ther. 10, 1109-1120. doi: 10.1016/j.ymthe.2004.08.016

Ng, E. S., Azzola, L., Bruveris, F. F., Calvanese, V., Phipson, B., Vlahos, K., et al. (2016). Differentiation of human embryonic stem cells to HOXA(+) hemogenic vasculature that resembles the aorta-gonad-mesonephros. Nat. Biotechnol. 34, 1168-1179. doi: $10.1038 /$ nbt.3702 
Ramos-Mejia, V., Navarro-Montero, O., Ayllon, V., Bueno, C., Romero, T., Real, P. J., et al. (2014). HOXA9 promotes hematopoietic commitment of human embryonic stem cells. Blood 124, 3065-3075. doi: 10.1182/blood-2014-03558825

Real, P. J., Ligero, G., Ayllon, V., Ramos-Mejia, V., Bueno, C., GutierrezAranda, I., et al. (2012). SCL/TAL1 regulates hematopoietic specification from human embryonic stem cells. Mol. Ther. 20, 1443-1453. doi: $10.1038 / \mathrm{mt} .2012 .49$

Real, P. J., Navarro-Montero, O., Ramos-Mejía, V., Ayllón, V., Bueno, C., and Menéndez, P. (2013). The role of RUNX1 isoforms in hematopoietic commitment of human pluripotent stem cells. Blood 121, 5250-5252. doi: 10.1182/blood-2013-03-487587

Sturgeon, C. M., Ditadi, A., Awong, G., Kennedy, M., and Keller, G. (2014). Wnt signaling controls the specification of definitive and primitive hematopoiesis from human pluripotent stem cells. Nat. Biotechnol. 32, 554-561. doi: 10.1038/ nbt. 2915

Wang, L., Li, L., Shojaei, F., Levac, K., Cerdan, C., Menendez, P., et al. (2004). Endothelial and hematopoietic cell fate of human embryonic stem cells originates from primitive endothelium with hemangioblastic properties. Immunity 21, 31-41. doi: 10.1016/j.immuni.2004.06.006

Wang, L., Menendez, P., Shojaei, F., Li, L., Mazurier, F., Dick, J. E., et al. (2005). Generation of hematopoietic repopulating cells from human embryonic stem cells independent of ectopic HOXB4 expression. J. Exp. Med. 201, 1603-1614. doi: $10.1084 /$ jem. 20041888

Conflict of Interest: The authors declare that the research was conducted in the absence of any commercial or financial relationships that could be construed as a potential conflict of interest.

Copyright (C) 2021 Gutierrez-Agüera, Rodriguez-Cortez, Petazzi, Bueno and Menendez. This is an open-access article distributed under the terms of the Creative Commons Attribution License (CC BY). The use, distribution or reproduction in other forums is permitted, provided the original author(s) and the copyright owner(s) are credited and that the original publication in this journal is cited, in accordance with accepted academic practice. No use, distribution or reproduction is permitted which does not comply with these terms. 